\title{
MRBLE-pep measurements reveal accurate binding affinities for B56, a PP2A regulatory subunit.
}

\author{
Jamin B. Hein ${ }^{1,2,3}$, Martha S. Cyert ${ }^{1}$, Polly M. Fordyce $2,4,5,6$ \\ ${ }^{1}$ Department of Biology, Stanford University, Stanford, CA 94305 \\ 2 Department of Bioengineering, Stanford University, Stanford, CA 94305 \\ ${ }^{3}$ The Novo Nordisk Foundation Center for Protein Research, Faculty of Health and Medical Sciences, University of Copenhagen, \\ Blegdamsvej 3b, 2200 Copenhagen, Denmark \\ 4 Department of Genetics, Stanford University, Stanford, CA 94305 \\ ${ }^{5}$ ChEM-H Institute, Stanford University, Stanford, CA 94305 \\ ${ }^{6}$ Chan Zuckerberg Biohub, San Francisco, CA 94110
}

\begin{abstract}
Signal transduction pathways rely on dynamic interactions between protein globular domains and short linear motifs (SLiMs). The weak affinities of these interactions are essential to allow fast rewiring of signaling pathways and downstream responses, but pose technical challenges for interaction detection and measurement. We recently developed a technique (MRBLE-pep) that leverages spectrally encoded hydrogel beads to measure binding affinities between a single protein and 48 different peptide sequences in a single small volume. In prior work, we applied it to map the binding specificity landscape between calcineurin and the PxIxIT SLiM (Nguyen et al. 2019). Here, using peptide sequences known to bind the PP2A regulatory subunit B56, we systematically compare affinities measured by MRBLE-pep or isothermal calorimetry (ITC) and confirm that MRBLE-pep accurately quantifies relative affinity over a wide dynamic range while using a fraction of the material required for traditional methods such as ITC.

\section{Introduction}

Cellular signaling transduction pathways are essential for every living organism. At the molecular level, many critical interactions within these pathways involve a globular protein domain binding a short (3-10 amino acid) linear motif (SLiM) within an intrinsically disordered region of a target protein (Neduva and Russell 2006; Dinkel et al. 2016; Davey, Cyert, and Moses 2015). These protein-protein interactions (PPIs) are considered attractive therapeutic targets, and there has been significant recent interest in developing small molecules to specifically target these PPI domains (e.g. PROTACS) (Paiva and Crews 2019). However, many protein-SLiM interactions remain either uncharacterized or poorly characterized, as they tend to be relatively weak ( $K_{\mathrm{d}} \mathrm{S}$ in the $1-10 \mu \mathrm{M}$ range) and are often dynamically regulated via reversible posttranslational modifications that modulate binding affinity to alter downstream responses, which complicates their detection, measurement, and characterization. New technologies for discovering, validating, and characterizing protein-SLiM interactions are therefore essential to unlock their therapeutic potential.

High-throughput technologies such as affinity purification coupled to mass spectrometry (Hertz et al. 2016), phage display (Blikstad and Ivarsson 2015; Ueki et al. 2019), and yeast two-hybrid assays (Mu et al. 2014), among others, have dramatically enhanced the pace of candidate protein-SLiM interaction discovery. Furthermore, computational modelling of motif determinants can allow in silico screening of entire proteomes to determine motif candidates (Krystkowiak, Manguy, and Davey 2018). However, these highthroughput methods generate large numbers (100s-1000s) of candidate interactors, many of which may be false positives. Validating and quantifying these low affinity binding interactions remains an experimental bottleneck, typically requiring sample- and labor-intensive low-throughput techniques such as isothermal calorimetry (ITC), surface plasmon resonance (SPR), and fluorescence polarization anisotropy, all of which require large amounts of purified protein.
\end{abstract}


In recent years, several technologies have attempted to bridge this gap between high-throughput, qualitative assays and low-throughput, quantitative assays. Protein microarrays (Meyer et al. 2018) and Luminex bead-based assays (Cook et al. 2019) allow multiplexed measurements of $\sim 10-1000$ interactions in parallel, but do not take place at equilibrium and therefore cannot return thermodynamic binding constants. Microscale thermophoresis and hold-up assay approaches can facilitate such thermodynamic measurements and have successfully been applied towards a variety of systems, including PDZ- and Chromo domain peptide interactions, antibody/antigen interactions, and receptor/ligand interactions (Wienken et al. 2010; Plach, Grasser, and Schubert 2017; Vincentelli et al. 2015). However, they still require fairly high amounts of purified protein.

To complement these approaches, we previously developed MRBLE-pep, a technology that leverages spectrally encoded beads for medium-throughput quantitative measurement of protein-peptide interactions using very small amounts of material ( $2 \mu \mathrm{mol}$ in $200 \mu \mathrm{L}$ for one concentration experiment, or $\sim 25 \mu \mathrm{g}$ for B56, the protein analyzed here). To create spectrally encoded beads, we use a microfluidic device to generate porous PEG-DA hydrogel droplets containing specific ratios of 4 different species of lanthanide nanophosphors (LNPs), each of which comprises a unique spectral code (MRBLEs, for Microspheres with Ratiometric Barcode Lanthanide Encoding) (Gerver et al. 2012; Nguyen et al. 2016) Using a custom-built fraction collector (Longwell and Fordyce 2020), we then direct all beads containing a given code to a specified well within a standard multi-well plate (Fig. 1A). After collection, beads are chemically functionalized with Fmoc-glycine and then transferred to a high-throughput peptide synthesizer for Fmoc solid phase peptide synthesis, yielding MRBLE-pep libraries with a unique 1:1 linkage between peptide sequence and embedded spectral code (Fig. 1A). After synthesis, MRBLE-pep bead libraries are pooled and incubated with a fluorescently-labeled protein of interest, followed by imaging to quantify protein binding and identify embedded codes (and thus, the underlying peptide sequence) (Fig. 1B). By repeating MRBLEpep measurements over multiple protein concentrations, we generate concentration-dependent binding curves, which are fitted to Langmuir isotherms to quantify interaction affinities.

In prior work, we applied MRBLE-pep to probe binding interactions between human calcineurin (CN), the $\mathrm{Ca} 2+/$ calmodulin regulated phosphatase and target of immunosuppressant drugs (Roy and Cyert 2020), and systematic mutations within the known PxIXIT SLiM. CN affinities for six PxIxIT peptide sequences correlated with those previously measured via orthogonal techniques and successfully predicted the strength of signaling inhibition in cells (Nguyen et al. 2019). However, these affinity comparisons were limited, and included several peptides with widely varying affinities reported in the literature. Unfortunately, our own attempts to acquire comparable data for these peptides using established technologies were unsuccessful.

Here, we extend the capabilities of the MRBLE-pep assay and further validate MRBLE-pep-derived affinity measurements using B56, a regulatory subunit of protein phosphatase 2A (Fig. 1C) (Ruvolo 2016; McCright and Virshup 1995). In recent work, the Nilsson group and others established that PP2A-B56 substrate specificity relies on a protein-protein interaction between B56 and the LxxIXE SLiM found in various substrates including KIF4A (a kinesin motor protein important for chromosome segregation) and FOXO3 (a transcription factor important for processes such as apoptosis and autophagy) (Fig. 1D) (Hertz et al. 2016; Wu et al. 2017). As differences in affinity between B56 and different substrates are thought to dictate the strength and relative timing of downstream signaling responses, the authors quantified the effects of different amino acid substitutions on binding affinities $\left(K_{d} S\right)$ for 8 LxxIxE SLiMs via ITC, revealing a previously unanticipated mode of substrate selection by B56. This set of 50 affinities with a wide dynamic range (spanning from 1-100 $\mu \mathrm{M}$ ), all measured by a single researcher expert in ITC, provide an ideal comparison set for testing the ability of MRBLE-pep to accurately return affinity information. By directly comparing 
MRBLE-pep- and ITC-derived affinities for B56 protein interacting with nine distinct peptides, we demonstrate that the MRBLE-pep measurements are in good agreement with ITC literature data. Importantly, the rank order of affinities between different peptides is preserved in MRBLE-pep measurements resulting in the same conclusions in terms of the binding sequence variations while requiring significantly less material.

\section{Results}

To test if MRBLE-pep can return accurate affinity information for an additional PPI system that also requires a different mode of protein detection, we measured binding of purified untagged B56 protein (graciously provided by the Nilsson lab (Hertz et al. 2016)) to two libraries of peptides (containing 22 and 24 peptides, respectively) via MRBLE-pep. To facilitate direct comparison between MRBLE-pep and ITC measurements over a wide dynamic range, each library included 9 peptides with previously measured affinities for B56 spanning 1 to $92 \mu \mathrm{M}$ (Fig. 1D).

A generalizable method for fluorescent detection of MRBLE-bound protein. Unlike in the original MRBLEpep publication (Nguyen et al. 2019) where we detected binding of His-tagged CN to libraries of MRBLEbound peptides via binding of a fluorescently-labeled anti-His antibody, here we detected binding of untagged B56 protein using a commercially-available anti-B56 primary antibody and an Alexa-647-labeled secondary antibody (Fig. 2A,B). Fluorescence images of bead-bound protein revealed a strong beadassociated Alexa-647 signal only in the presence of the B56 protein, establishing that non-specific antibody binding to MRBLE-bound peptides was insignificant (Fig. 2A,B). Fluorescent signals for beads coupled to known peptides with high affinity for B56 were $>30$-fold higher than those for negative controls, establishing that binding was detected with a high dynamic range (Fig. 2A,B).

Estimating time-to-equilibrium and verifying appropriate concentrations. Accurately estimating interaction affinities by fitting Langmuir isotherms to observations of concentration-dependent binding relies on two main assumptions. First, interactions must have reached steady state, with no further change in the relative bound and unbound protein fractions over time. Second, the amount of protein ligand available for binding must be in vast excess of the amount of MRBLE-bound peptides in each experiment and significantly higher than expected interaction $K_{d} S$ (Pollard 2010). For protein-peptide interactions (PPIs) in solution, the time required for a given $\mathrm{PPI}$ to reach equilibrium $\left(K_{\text {eq }}\right)$ can be loosely approximated by the dissociation rate of the interaction $\left(k_{\text {off }}\right)$. For relatively weak interactions ( $K_{\mathrm{d}} \mathrm{S}$ in the 1-10 $\mu \mathrm{M}$ range), this dissociation rate typically ranges from $\sim 10^{3}-10^{5} / \mathrm{s}$, corresponding to a time to reach equilibrium of $\sim 0.04-4 \mathrm{~s}$ (Jarmoskaite et al. 2020). In our prior MRBLE-pep experiments, the time required for CN-peptide interactions to reach equilibrium was approximately 14 hours, presumably due to the fact that $\mathrm{CN}$ proteins interacting with peptides covalently coupled to the hydrogel matrix were sterically constrained within the porous hydrogel mesh, where the high local concentration of peptide ligands promotes rebinding (Fig. 2B). These slow equilibration times are critical for assay performance, as they make it possible to wash and image beads without loss of weakly bound material during imaging. However, it remained unclear if additional proteins would also require extended times to reach assay equilibrium or whether this property would depend on additional factors such as peptide density, buffer composition, or the identity of the protein itself.

To experimentally test the time required for MRBLE-pep B56 binding to reach equilibrium, we incubated B56 protein at $2 \mu \mathrm{M}$ with a MRBLE-bound peptide sequence with high affinity for B56 (TSFFSGLSPIEEEAD, code 10) (Fig. S1-4). The reactions required 24 hours to reach equilibrium (Figs. 3C,S1-4), providing additional support for a model in which protein/peptide dissociation rates are significantly slowed by protein rebinding to other peptides immobilized within the hydrogel matrix. Based on these measurements, we quantified binding for all subsequent B56 MRBLE-pep experiments after 24 hours. 
To confirm that B56 protein was indeed in vast excess, we first calculated the expected concentrations of B56 protein and available peptides within each binding assay. For a given peptide sequence, we use approximately 100 beads with an estimated peptides density of $10^{8}$ peptides per bead (Nguyen et al. 2019). We therefore have a peptide concentration of $2.4 \times 10^{11}$ peptides in a $100 \mu \mathrm{l}$ assay volume for 24 different codes and a protein concentration of $1.86 \times 10^{13}$ at the lowest concentration of protein (31 nmol). For direct experimental validation, we additionally removed the supernatant after 24 hours of incubation and quantified remaining B56 via Western blot, establishing no depletion over the full range of concentrations tested (Fig. S5).

Comparing affinities measured across MRBLE-pep technical replicates. Next, for each MRBLE-pep library, we performed 3 technical replicate experiments measuring concentration-dependent binding for B56 protein at 7 concentrations ranging from 31 to $2000 \mathrm{nmol}$, then globally fit the median intensities for all beads containing a given code at each concentration to a Langmuir isotherm (Figs. 4A, S6-13):

Eqn. 1:

$$
I_{i}=\frac{I_{\max } *[B 56]}{[B 56]+K_{d, i}}
$$

Here, $I_{i}$ represents the median fluorescence signal associated with all beads bearing a particular peptide at a given concentration, $I_{\max }$ is a global saturation value shared across all peptides, [B56] represents the soluble B56 concentration available for binding, and $K_{\mathrm{d}, \mathrm{i}}$ is the binding affinity for a given peptide. By constraining all curves to share a single saturation value for fluorescence intensity, this global fitting procedure returns reliable binding affinity estimates even for peptides with $K_{d} S$ well above the maximum B56 concentration used in the assay (see Materials and Methods). We then convert measured $K_{\mathrm{d}} \mathrm{S}$ (absolute affinities) to relative differences in binding energies upon mutation ( $\Delta \Delta \mathrm{Gs})$ using the following equation:

Eqn. 2:

$$
\Delta \Delta G_{i}=R T * \ln \left(K_{d, i} / K_{d, r e f}\right)
$$

Here, $\Delta \Delta \mathrm{G}_{\mathrm{i}}$ represents the relative change in binding energy for a given peptide with $K_{\mathrm{d}, \mathrm{i}}$ relative to a 'reference' peptide and $R$ and T represent the natural gas constant and temperature, respectively. Pairwise comparisons of measured $K_{d}$ and $\Delta \Delta G$ values between replicates for both libraries established that measurements were highly reproducible, with $r^{2}$ values of 0.73-0.99 and RMSEs of 0.11-0.37 for $K_{\mathrm{d}} \mathrm{S}$ ranging over nearly 3 orders of magnitude and $r^{2}$ values of 0.95-0.98 and RMSEs of 0.03-0.65 for $\Delta \Delta$ Gs spanning nearly $5 \mathrm{kcal} / \mathrm{mol}$ (Figs. S9,S13). Measurements for the same peptide across the two different MRBLE-pep libraries also showed strong agreement $\left(r^{2}=0.86\right.$ and RMSE $=0.84$ for $K_{d}$ comparisons) (Figs. 4B). Overall, measured affinities for peptides from library \#2 were systematically higher than for library \#1, consistent with prior observations that uncertainties in the estimated intensity at saturation can lead to systematic variations in measured affinities. However, relative differences in binding energies remained consistent, particularly when considering systematic mutations within a given peptide backbone $\left(r^{2}=0.83\right.$ and RMSE $=0.59$ for $\Delta \Delta \mathrm{G}$ comparisons, corresponding to $<2$-fold differences across experiments).

Comparing affinities measured via MRBLE-pep and ITC. Next, we sought to directly compare affinities measured via MRBLE-pep and ITC (isothermal calorimetry). While ITC has traditionally been considered the 'gold standard' method for measuring $K_{\mathrm{d}} \mathrm{S}$ for protein interactions, these experiments require large amounts of highly purified protein, particularly for weak protein-peptide interactions (900 $\mu \mathrm{g}$ per protein/peptide interaction for $K_{d} S$ in the low micromolar range). MRBLE-pep measurements require significantly less material ( $25 \mu \mathrm{g}$ for 22 protein/peptide interaction measurements, or $1 \mu \mathrm{g}$ per interaction) and labor (as multiple protein/peptide interactions can be assessed in a single experiment), but to date have only been shown to return thermodynamic binding information for a single protein (CN).

Measured affinities $\left(K_{d} S\right)$ for B56 binding to 12 peptides containing systematic substitutions within the LxxIxE motif found within Kif4A or FoxO3 showed strong correlation between the two methods $\left(r^{2}=0.83\right.$ 
and 0.70 for libraries 1 and 2, respectively) (Fig. 5A). Measured affinities from library \#1 showed better agreement with ITC-derived affinities (RMSE $=0.37$ and 0.61 for libraries 1 and 2, respectively), largely due to a single measurement for a $\mathrm{FoxO} 3$ variant containing additional negatively charged residues at the $\mathrm{C}$ terminal end of the sequence (LRQSPMTIQEEEPA) (Fig. 5B, Table S1). Overall, library 2 measurements returned systematically higher affinities, potentially due to small variations in the surface peptide density of displayed peptides between replicates. However, measurements of relative changes in binding affinities upon mutation $(\Delta \Delta G s)$ showed strong correlation for both libraries $\left(r^{2}=0.93\right.$ and 0.94 for both libraries, with RMSEs of 0.48 and 0.31 , respectively, and the rank order of preferred sequences remained largely unchanged. Together, these comparisons establish that MRBLE-pep returns binding affinities with similar accuracy to ITC but using 1/500th of the material.

Applying MRBLE-pep to investigate how mutations within LXXIXE SLiMs affect B56 binding. Having established the accuracy of MRBLE-pep, we applied it to investigate the effects of systematic substitutions within the LxxIXE SLiM motifs present within Kif4A, FoxO3, GEF-H1, and Rac-GAP1. In particular, we investigated the influence of different amino acids in the first position and those surrounding the acidic last position of the motif. In addition, we probed how phosphorylation at different positions in close proximity to key positions affected binding affinities. (Fig. 6). Within Kif4A, the LxxIxE motif appears as a CxxIxE sequence (Fig. 6A). As expected, making a $C$ to $L$ substitution at the first position within the SLiM (which alters the site to look more like the known consensus) enhances affinity by $\sim 2.5 \mathrm{kcal} / \mathrm{mol}$ (Fig. 6A). Overall, the $L$ residue is strongly preferred at this position, with $F$ and $M$ substitutions also moderately enhancing binding relative to the native $\mathrm{C}$. In the presence of mutations to this first position, mutating the additional $I$ and $E$ conserved residues reduces binding to background levels. Surprisingly, substituting the $L$ or the $E$ position with a $\mathrm{Y}$ in a high affinity Lxx|xE motif did not completely abolish binding (reduction of $\sim 2$ or $\sim 3$ $\mathrm{kcal} / \mathrm{mol}$, respectively), suggesting that SLiMs containing an $\mathrm{L}$ in this first position or multiple acidic residues at the C-terminal boundary may be able to tolerate substitutions at other conserved positions while maintaining biological function (Fig 6A). However, future in vivo work is required to test this hypothesis.

In recent work, the Nilsson lab also reported that phosphorylation generally increases B56 binding affinities when adjacent to either the glutamic acid residues at the C-terminus of the LxxIxE SLiM or to the conserved $\mathrm{N}$-terminal leucine. Here, we find that the effects of phosphorylation are strongly context-dependent: while a single phosphorylated residue adjacent to the conserved leucine leads to slightly enhanced affinities, the addition of a second phosphorylated residue at the C-terminus of the Kif4A SLiM dramatically decreases binding (Fig. 6A). By contrast, the addition of a phosphorylated serine $\mathrm{N}$-terminal to the FoxO3 LxxIxE motif reduces binding (Fig. 6B), phosphorylation of residues adjacent to the glutamic acids within the GEF-H1 SLiM has no effect on affinities, and phosphorylation immediately C-terminal to the Rac-GAP1 LxxIxE SLiM dramatically enhances binding (Fig. 6C,D). Interestingly, an additional $E$ after the obligatory $E$ seems to increase affinity more than a phosphorylated serine (Fig. 6D). Furthermore, we observed a strong decrease in binding affinity when mutating position five ( $(\mathrm{xx} \mid \mathbf{x} E)$ from $D$ to $G$ in FoxO3, indicating that this position could be important for fine tuning the affinity between B56 and its binding partners (Fig. 6B); future experiments are required to systematically probe how substitutions at this position alter affinity. Together, these results highlight the importance of local sequence context in determining binding strength, an observation further supported by the fact that $M$ to $L$ substitutions within a variety of different peptide backbones have different effects (Fig. 6E).

\section{Discussion}

In prior work, we described the development of the MRBLE-pep assay and demonstrated that MRBLE-pep was able to quantitatively profile the binding specificity landscape for $\mathrm{CN}$ interacting with a library of $\sim 400$ peptides containing systematic substitutions within the PxIXIT SLiM. Here, we further validate that MRBLEpep agrees with previous ITC measurements in reporting the relative effects of amino acid substitutions on 
binding affinities of an additional SLiM (LXXIXE) for its receptor (B56), while using a fraction of the required material and requiring a fraction of the time. Moreover, we establish that protein binding can be accurately quantified using an unlabeled primary antibody and fluorescently-labeled secondary antibody, expanding the capabilities of the assay to examine proteins that are difficult to tag and/or purify.

While technical replicates using independent MRBLE-pep libraries showed systematic shifts in raw affinities

(Fig. 5), we establish that measured $\Delta \Delta G s$ and the rank order of peptide sequence preferences are highly reproducible. We speculate that these systematic shifts may result from differences in the density of displayed peptides after on-bead synthesis. In recent work, we described a dramatically simplified method for encoded bead production and functionalization (MRBLEs 2.0) (Feng et al. 2020), and we anticipate that peptide synthesis on beads produced via this new pipeline will yield more consistent density coverage. Furthermore, by simplifying the technology we hope that these improvements will allow researchers to more broadly adopt MRBLE-pep to systematically characterize SLiM binding determinants and to validate candidate interactions identified by high-throughput phage-display, yeast-two-hybrid or in silico screens.

\section{Materials and Methods}

MRBLE production and peptide synthesis. We produced MRBLEs spectrally encoded hydrogel beads as described previously (Hein et al. 2020; Nguyen et al. 2019).

B56 expression and purification. B56 was a kind gift from the lab of Jakob Nilsson and was expressed and purified as described previously (Hertz et al. 2016) .

MRBLE-pep time-to-equilibrium assays. To reduce nonspecific binding, we 'blocked' beads by incubating approximately 500 beads per code with PBST (0.1\% Tween-20) and $5 \%$ BSA for 1 hour at RT. To allow labeled antibody to bind B56 protein prior to bead assays, we preincubated $2 \mu$ mol recombinant B56 protein with $1 \mu \mathrm{mol}$ mouse anti-B56 antibody (Clone 23/B56 $\alpha$ BD biosciences, 610615) and $1 \mu \mathrm{mol}$ goat anti-mouse IgG Alexa Fluor 647 (Thermo Fisher A-21235) in $500 \mu \mathrm{L}$ binding buffer (50 mM Tris $\mathrm{pH}=7.5$, $150 \mathrm{mM} \mathrm{NaCl}, 0.1 \%$ TWEEN 20) for one hour at $4{ }^{\circ} \mathrm{C}$. Prior to beginning binding assays, we washed beads once with binding buffer and divided them into five $200 \mu$ l Eppendorf tubes. After decanting the binding buffer, we added $100 \mu \mathrm{L}$ of the B56 protein and anti-B56 and anti-mouse IgG antibody complex. At specified times, we removed protein-antibody complexes, washed beads once with $100 \mu \mathrm{L}$ binding buffer, and resuspended in $20 \mu \mathrm{L}$ PBST prior to imaging.

Western blotting to test for depletion of B56 after binding assays. Each gel electrophoresis reaction used 6 $\mu \mathrm{L}$ of unbound B56 protein- antibody complex and $2 \mu \mathrm{L}$ of $4 \times$ SDS-PAGE sample buffer (Thermo Fisher). After heating the samples to $95 \mathrm{C}$ for 10 minutes, we loaded samples on a SDS-PAGE gel (4-20\% Precast Protein Gels, Bio-Rad \#4561095DC) and separated the protein at $100 \mathrm{~V}$ for $60 \mathrm{~min}$. We then blotted the separated proteins onto a PVDF membrane for $90 \mathrm{~min}$ at $400 \mathrm{~mA}$ using a Bio-Rad wet blotting system. The membrane was blocked with $3 \%$ BSA in TBS-T for 30 min at room temperature with gentle agitation. We removed the buffer and added fresh TBS-T with 3\% BSA and Goat anti-Mouse IR-Dye 700 (1:5000 diluted) to obtain a background signal from the Mouse anti-B56 antibody present in the samples. After 2 hours incubation with gentle agitation at room temperature, we washed the blot three times for five minutes with TBS-T, dried, and scanned using the Odyssey CLx imaging system (Licor Biosciences). The membrane was incubated again with $3 \%$ BSA in TBS-T for 30 min at room temperature. The buffer was exchanged and $500 \mathrm{ng}$ of Mouse anti-B56 antibody was added and incubated for 2 hours at room temperature. The membrane was again washed 3 times for 5 minutes with TBS-T and then we added fresh buffer containing 1:5000 diluted Goat anti-Mouse IR-Dye 700 and incubated for 1 hour at room temperature. After additional washing, the membrane was scanned using the Odyssey CLx imaging system. 
MRBLE-pep concentration-dependent binding assays. Each concentration-dependent binding assay used approximately 700 beads per code. To begin the assay, we blocked beads with PBST (0.1\% Tween-20) and $5 \%$ BSA for 1 hour at RT to reduce non-specific binding. During this blocking step, we also preincubated $200 \mu \mathrm{L}$ of $2 \mu \mathrm{mol}$ recombinant B56 protein with $1 \mu \mathrm{mol}$ mouse anti-B56 antibody (Clone 23/B56a BD biosciences, 610615) and $1 \mu \mathrm{mol}$ goat anti-mouse IgG Alexa Fluor 647 (Thermo Fisher A-21235) for one hour at 4 degrees to form B56/antibody complexes. Next, we washed beads once with $700 \mu$ l binding buffer and then divided them into seven $200 \mu$ Eppendorf tubes to yield approximately 100 beads per code per assay. B56/antibody complexes were diluted from $2 \mu \mathrm{mol}$ to the appropriate assay concentration using binding buffer containing $10 \%$ glycerol and added to Eppendorf tubes containing beads to a final reaction volume of $100 \mu \mathrm{L}$. After 24 hours, we removed the unbound protein-antibody complexes, washed beads once with $100 \mu \mathrm{L}$ of binding buffer, and resuspended in $20 \mu \mathrm{L}$ PBST.

MRBLE-pep imaging. We imaged all beads exactly as previously described (Nguyen et al. 2019; Hein et al. 2020; Harink et al. 2019). At the end of this analysis, we quantified a median intensity for all pixels associated with this bead annulus for each bead [source data].

Global fitting of concentration-dependent binding curves. To generate binding curves for each code, we first plotted the median of all median bead annulus fluorescence intensities as a function of soluble B56 concentration. To determine the $K_{d}$ values for each protein/peptide interaction, we then globally fit the data for all codes to a single-site binding model(Maerkl and Quake 2007; Fordyce et al. 2010; Aditham et al. 2020) :

$$
I_{i}[B 56]=\frac{I_{\max } *[B 56]}{[B 56]+K_{d, i}}
$$

Here, li[B56] denotes the measured bead-associated Cy5 intensity as a function of soluble B56 concentration for a given code, Imax is a global constant shared across all codes corresponding to the maximum bead-associated Cy5 intensity at saturating [B56], [B56] denotes the concentration of soluble B56 available for binding, and $K \mathrm{~d}$, i denotes the dissociation constant $(K \mathrm{~d})$ for a given B56-peptide complex. This global fitting procedure assumes that: (1) the binding interaction is measured at steady-state, and (2) the stoichometry of binding remains constant across all B56-peptide interactions (making it possible to estimate $K_{d} S$ even for very weak interactions with $K_{d}$ values greater than the maximum soluble B56 concentration probed in the assay.

To calculate $\Delta \Delta G$ s across a given experiment, we used the following formula:

$$
\Delta \Delta G_{i}=R T * \ln \left(K_{d, i} / K_{d, r e f}\right)
$$

where $\mathrm{R}$ is the gas constant $\left(1.987 \cdot 10^{-3} \mathrm{kcal} /(\mathrm{K} \cdot \mathrm{mol})\right), \mathrm{T}$ is $298 \mathrm{~K}$, and $K_{\mathrm{d}, \mathrm{i}}$ is the median of the returned $K_{d} S$ from technical replicates of concentration-depending binding experiments for a protein-peptide interaction of interest and $K_{\mathrm{d} \text {,ref }}$ is the median of the returned $K_{\mathrm{d}} \mathrm{S}$ from technical replicates of concentrationdepending binding experiments for a reference protein-peptide interaction.

Comparing MRBLE-pep technical replicates for a given library. To compare measurements between technical replicates using a given MRBLE-pep library, we performed pairwise comparisons between each replicate and evaluated concordance via a linear regression of: (1) $\log _{10}$-transformed $K_{\mathrm{d}}$ values for each peptide returned from the global fitting procedure described above, and (2) calculated $\Delta \Delta \mathrm{G}$ values relative to a single reference peptide. 
Comparing MRBLE-pep measurements across libraries. To compare measurements across MRBLE-pep libraries, we directly compared: (1) the median $\log _{10}$-transformed $K_{d}$ value from the 3 technical replicates of each library and (2) $\Delta \Delta G$ values calculated from these median $K_{d}$ values. In each case, we again evaluated agreement via a linear regression.

Comparing MRBLE-pep measurements with ITC. To compare measurements between MRBLE-pep and ITC, we again directly compared the median $\log _{10}$-transformed $K_{d}$ value from the 3 technical replicates of each library with prior measurements from the Nilsson lab (Hertz et al. 2016).

\section{Acknowledgements}

P.M.F. and M.S.C. gratefully acknowledge the support of a Stanford Bio-X Interdisciplinary Initiatives Seed Grant. This work has been supported by NIH grant 1DP2GM123641 awarded to P.M.F., and P.M.F. is also a Chan Zuckerberg Biohub Investigator. JBH was funded by grant NNF170C0025404 from the Novo Nordisk Foundation and the Stanford Bio-X Program. M.S.C. is funded by NIH grant R35GM136243. P.M.F. previously co-authored a patent describing production of spectrally encoded beads via ratiometric barcode lanthanide encoding (WO 2014/031902 A2). 


\section{References}

Aditham, Arjun, Craig Markin, Daniel Mokhtari, Nicole DelRosso, and Polly Fordyce. 2020. "HighThroughput Binding Affinity Measurements for Mutations Spanning a Transcription Factor-DNA Interface Reveal Affinity and Specificity Determinants." BioRxiv. https://doi.org/10.1101/2020.06.22.165571.

Blikstad, Cecilia, and Ylva Ivarsson. 2015. "High-Throughput Methods for Identification of Protein-Protein Interactions Involving Short Linear Motifs." Cell Communication and Signaling : CCS 13 (2015): 38. https://doi.org/10.1186/s12964-015-0116-8.

Cook, Damon B., Brian C. McLucas, Leticia A. Montoya, Chris M. Brotski, Shelley Das, Markus Miholits, and Thao H. Sebata. 2019. "Multiplexing Protein and Gene Level Measurements on a Single Luminex Platform." Methods 158 (January): 27-32. https://doi.org/10.1016/j.ymeth.2019.01.018.

Davey, Norman E, Martha S Cyert, and Alan M Moses. 2015. "Short Linear Motifs - Ex Nihilo Evolution of Protein Regulation." Cell Communication and Signaling : CCS 13 (1): 43. https://doi.org/10.1186/s12964-015-0120-z.

Dinkel, Holger, Kimvan Roey, Sushama Michael, Manjeet Kumar, Bora Uyar, Brigitte Altenberg, Vladislava Milchevskaya, et al. 2016. "ELM 2016 - Data Update and New Functionality of the Eukaryotic Linear Motif Resource." Nucleic Acids Research 44 (D1): D294-300. https://doi.org/10.1093/nar/gkv1291.

Feng, Yinnian, Adam White, Jamin Hein, Eric Appel, and Polly Fordyce. 2020. “MRBLES 2.0: HighThroughput Generation of Chemically Functionalized Spectrally and Magnetically-Encoded Hydrogel Beads Using a Simple Single-Layer Microfluidic Device," no. 650. https://doi.org/10.1101/2020.06.22.166074.

Fordyce, Polly M., Doron Gerber, Danh Tran, Jiashun Zheng, Hao Li, Joseph L. Derisi, and Stephen R. Quake. 2010. "De Novo Identification and Biophysical Characterization of Transcription-Factor Binding Sites with Microfluidic Affinity Analysis." Nature Biotechnology 28 (9): 970-75. https://doi.org/10.1038/nbt.1675.

Gerver, R. E., R. Gómez-Sjöberg, B. C. Baxter, K. S. Thorn, P. M. Fordyce, C. A. Diaz-Botia, B. A. Helms, and J. L. Derisi. 2012. "Programmable Microfluidic Synthesis of Spectrally Encoded Microspheres." Lab on a Chip 12 (22): 4716-23. https://doi.org/10.1039/c2lc40699c.

Harink, Björn, H Q Nguyen, Kurt Thorn, and Polly Fordyce. 2019. "An Open-Source Software Analysis Package for Microspheres with Ratiometric Barcode Lanthanide Encoding (MRBLEs)." PLOS ONE 14 (3). https://doi.org/10.1371/journal.pone.0203725.

Hein, Jamin, Huy Nguyen, Martha Cyert, and Polly Fordyce. 2020. "Protocol for Peptide Synthesis on Spectrally Encoded Beads for MRBLE-Pep Assays." Bio-Protocol 10 (13): 1-26. https://doi.org/10.21769/BioProtoc.3669.

Hertz, Emil P T, Thomas Kruse, Norman E Davey, Blanca Lopez Mendez, Jon Otti Sigurdsson, Guillermo Montoya, Jesper V Olsen, and Jakob Nilsson. 2016. "A Conserved Motif Provides Binding Specificity to A Conserved Motif Provides Binding Specificity to the PP2A-B56 Phosphatase." Molceular Cell, 686-95. https://doi.org/10.1016/j.molcel.2016.06.024.

Jarmoskaite, Inga, Ishraq Alsadhan, Pavanapuresan P. Vaidyanathan, and Daniel Herschlag. 2020. "How to Measure and Evaluate Binding Affinities.” ELife 9: 1-34. https://doi.org/10.7554/ELIFE.57264. 
Krystkowiak, Izabella, Jean Manguy, and Norman E Davey. 2018. "PSSMSearch: A Server for Modeling, Visualization, Proteome-Wide Discovery and Annotation of Protein Motif Specificity Determinants." Nucleic Acids Research 46 (W1): W235-41. https://doi.org/10.1093/nar/gky426.

Longwell, Scott A., and Polly M. Fordyce. 2020. "MicrlO: An Open-Source Autosampler and Fraction Collector for Automated Microfluidic Input-Output." Lab on a Chip 20 (1): 93-106. https://doi.org/10.1039/c9lc00512a.

Maerkl, Sebastian J., and Stephen R. Quake. 2007. "A Systems Approach to Measuring the Binding Energy Landscapes of Transcription Factors." Science 315 (5809): 233-37. https://doi.org/10.1126/science.1131007.

McCright, B, and D M Virshup. 1995. "Identification of a New Family of Protein Phosphatase 2A Regulatory Subunits." The Journal of Biological Chemistry 270 (44): 26123-28. https://doi.org/10.1074/jbc.270.44.26123.

Meyer, Katrina, Marieluise Kirchner, Bora Uyar, Jing-Yuan Cheng, Giulia Russo, Luis R. HernandezMiranda, Anna Szymborska, et al. 2018. "Mutations in Disordered Regions Can Cause Disease by Creating Dileucine Motifs." Cell 175 (1): 239-253.e17. https://doi.org/10.1016/j.cell.2018.08.019.

Mu, Yi, Pengfei Cai, Siqi Hu, Sucan Ma, and Youhe Gao. 2014. "Characterization of Diverse Internal Binding Specificities of PDZ Domains by Yeast Two-Hybrid Screening of a Special Peptide Library." PLOS ONE 9 (2). https://doi.org/10.1371/journal.pone.0088286.

Neduva, Victor, and Robert B Russell. 2006. "Peptides Mediating Interaction Networks : New Leads at Last." Current Opinion in Biotechnology, 465-71.

Nguyen, H Q, Brian C Baxter, Kara Brower, Camilo A Diaz-botia, Joseph L Derisi, Polly M Fordyce, and Kurt S Thorn. 2016. "Programmable Microfluidic Synthesis of Over One Thousand Uniquely Identifiable Spectral Codes." Advanced Optical Materials, 1-6.

Nguyen, H Q, Jagoree Roy, Björn Harink, Nikhil P Damle, Naomi R Latorraca, Brian C Baxter, Kara Brower, et al. 2019. "Quantitative Mapping of Protein-Peptide Affinity Landscapes Using Spectrally Encoded Beads." ELife 8 (July). https://doi.org/10.7554/eLife.40499.

Paiva, Stacey-Lynn, and Craig M Crews. 2019. "Targeted Protein Degradation: Elements of PROTAC Design." Current Opinion in Chemical Biology 50 (June): 111-19. https://doi.org/10.1016/J.CBPA.2019.02.022.

Plach, Maximilian, Klaus Grasser, and Thomas Schubert. 2017. "MicroScale Thermophoresis as a Tool to Study Protein-Peptide Interactions in the Context of Large Eukaryotic Protein Complexes." BioProtocol 7 (23): 1-24. https://doi.org/10.21769/bioprotoc.2632.

Pollard, Thomas D. 2010. "MBOC Technical Perspective: A Guide to Simple and Informative Binding Assays." Molecular Biology of the Cell 21 (23): 4061-67. https://doi.org/10.1091/mbc.E10-08-0683.

Roy, Jagoree, and Martha S. Cyert. 2020. "Identifying New Substrates and Functions for an Old Enzyme: Calcineurin." Cold Spring Harbor Perspectives in Biology 12 (3). https://doi.org/10.1101/cshperspect.a035436.

Ruvolo, Peter P. 2016. "The Broken 'Off' Switch in Cancer Signaling: PP2A as a Regulator of Tumorigenesis, Drug Resistance, and Immune Surveillance." BBA Clinical 6: 87-99. https://doi.org/10.1016/j.bbacli.2016.08.002. 
Tareen, Ammar, and Justin B. Kinney. 2020. "Logomaker: Beautiful Sequence Logos in Python." Bioinformatics 36 (7): 2272-74. https://doi.org/10.1093/bioinformatics/btz921.

Ueki, Yumi, Thomas Kruse, Melanie Bianca Weisser, Gustav N. Sundell, Marie Sofie Yoo Larsen, Blanca Lopez Mendez, Nicole P. Jenkins, et al. 2019. "A Consensus Binding Motif for the PP4 Protein Phosphatase." Molecular Cell 76 (6): 953-964.e6. https://doi.org/10.1016/j.molcel.2019.08.029.

Vincentelli, Renaud, Katja Luck, Juline Poirson, Jolanta Polanowska, Julie Abdat, Marilyne Blémont, Jeremy Turchetto, et al. 2015. "Quantifying Domain-Ligand Affinities and Specificities by High-Throughput Holdup Assay." Nature Methods 12 (8): 787-93. https://doi.org/10.1038/nmeth.3438.

Wienken, Christoph J., Philipp Baaske, Ulrich Rothbauer, Dieter Braun, and Stefan Duhr. 2010. "ProteinBinding Assays in Biological Liquids Using Microscale Thermophoresis." Nature Communications 1 (7). https://doi.org/10.1038/ncomms1093.

Wu, Cheng Guo, Hui Chen, Feng Guo, Vikash K. Yadav, Sean J. Mcllwain, Michael Rowse, Alka Choudhary, et al. 2017. "PP2A-B' Holoenzyme Substrate Recognition, Regulation and Role in Cytokinesis." Cell Discovery 3: 1-19. https://doi.org/10.1038/celldisc.2017.27. 
bioRxiv preprint doi: https://doi.org/10.1101/2020.12.16.423088; this version posted December 16, 2020. The copyright holder for this preprint (which was not certified by peer review) is the author/funder, who has granted bioRxiv a license to display the preprint in perpetuity. It is made available under aCC-BY-NC-ND 4.0 International license.

A spectrally encoded beads (48 codes)
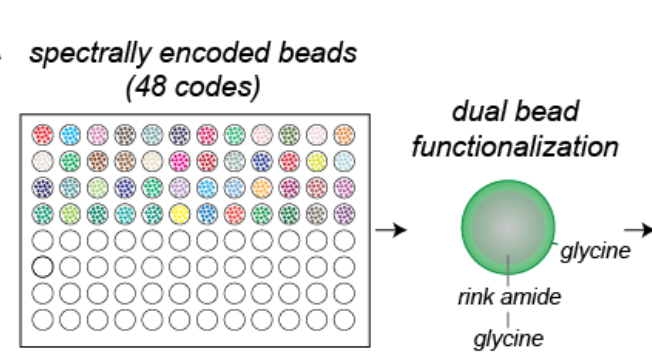

high-throughput peptide synthesis

bead-bound peptide library
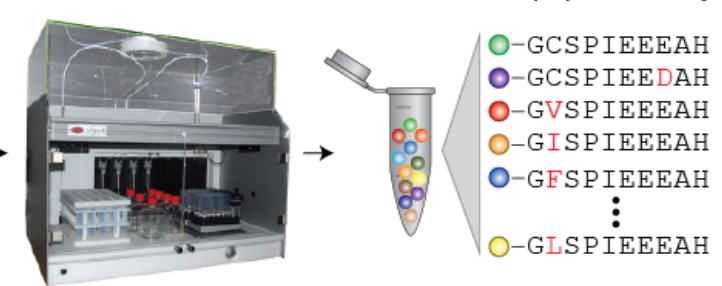

B MRBLE-pep binding assay
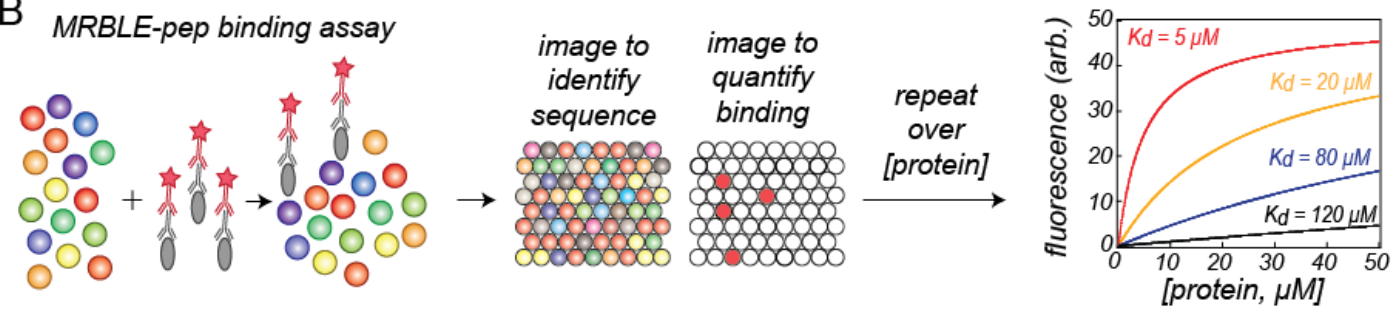

C

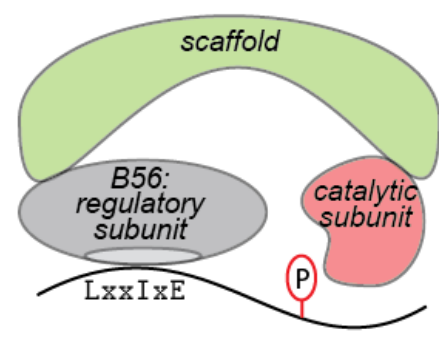

$\mathrm{D}$

ITC affinities from ref. $X X$ :

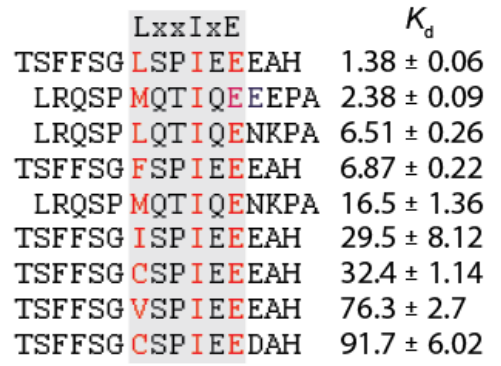

$E$

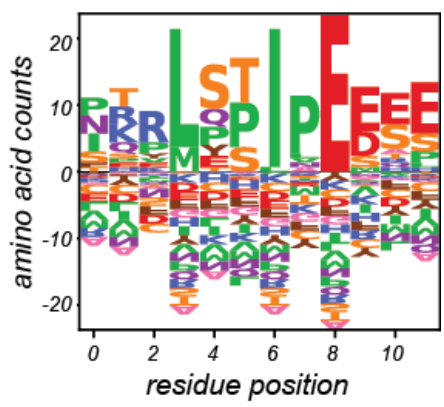

Figure 1. Overview of MRBLE-pep workflow and B56 specificity. (A) Schematic showing MRBLE-pep peptide library production. Spectrally encoded hydrogel beads containing unique ratios of lanthanide nanophosphors (LNPs) are functionalized with rink amide/glycine linkers within bead cores and glycine linkers in the outer shell; after functionalization, peptides are synthesized directly on beads with a 1:1 linkage between the peptide sequence identity and embedded spectral code via solid-phase peptide synthesis in a high-throughput peptide synthesizer. (B) MRBLE-bound peptide libraries are then pooled and incubated with a protein of interest, primary antibody, and labeled secondary antibody. After binding reactions reach steady state, beads are imaged in the lanthanide channels (to identify the peptide sequence associated with each bead) and in fluorescence channels (to quantify the amount of bead-bound protein). Measurements of binding at multiple concentrations can be globally fit to Langmuir isotherms to quantify interaction affinities. (C) Cartoon model of a the heterotrimeric B56-PP2A complex binding a substrate containing the LXXIXE SLiM recognition site. (D) Affinities measured by ITC for a set of 9 peptides containing systematic substitutions within the LxxIxE SLiMs from Kif4A and FoxO3 (data from REFERENCE). (E) Consensus position-specific specificity matrix generated from known B56 binding sites (data from http://slim.icr.ac.uk/pp2a/index.php?page=instances\#28884018) and (Hertz et al. 2016; Wu et al. 2017); logo generated using LogoMaker software (Tareen and Kinney 2020). 
bioRxiv preprint doi: https://doi.org/10.1101/2020.12.16.423088; this version posted December 16,2020 . The copyright holder for this preprint (which was not certified by peer review) is the author/funder, who has granted bioRxiv a license to display the preprint in perpetuity. It is made available under aCC-BY-NC-ND 4.0 International license.
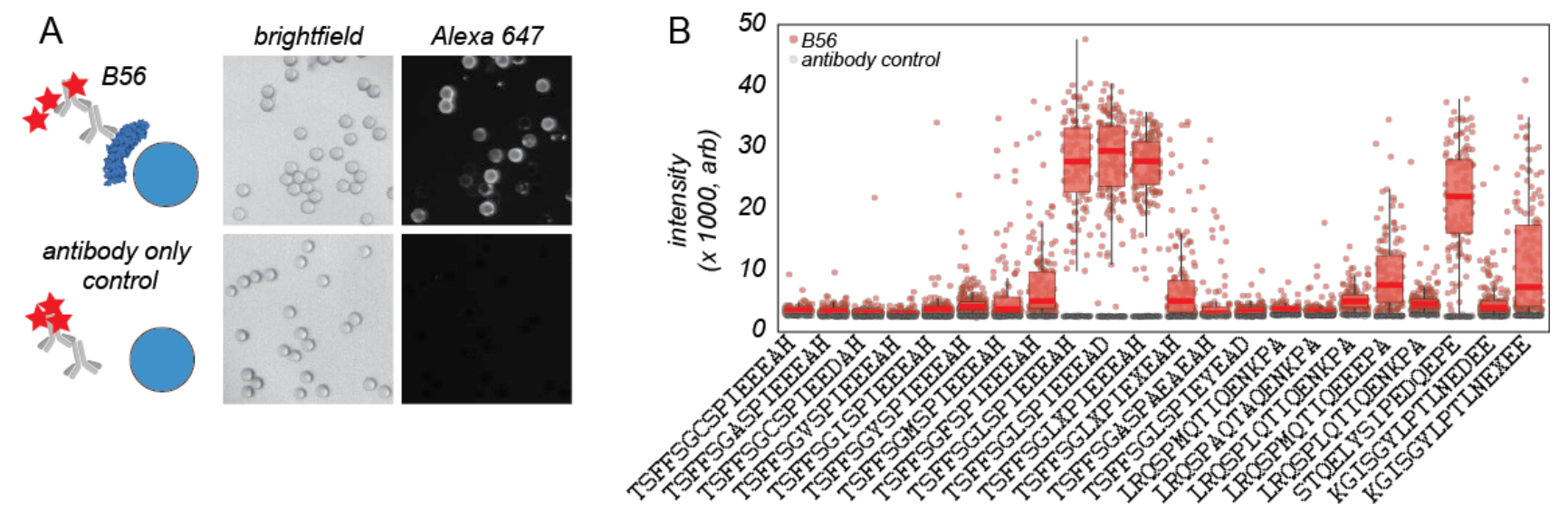

Figure 2. Example images and measured fluorescence intensities for beads incubated with antibodies and B56 or antibodies alone (negative control). (A) Schematic of binding assays and example images of beads in the bright field and Alexa 647 fluroescence channels. (B) Median binding intensities for each bead (markers) and 95\% confidence intervals (box plots) for binding assays with B56 and antibodies (red) or antibodies alone (grey) for a MRBLE-pep assay with 22 different peptide sequences.
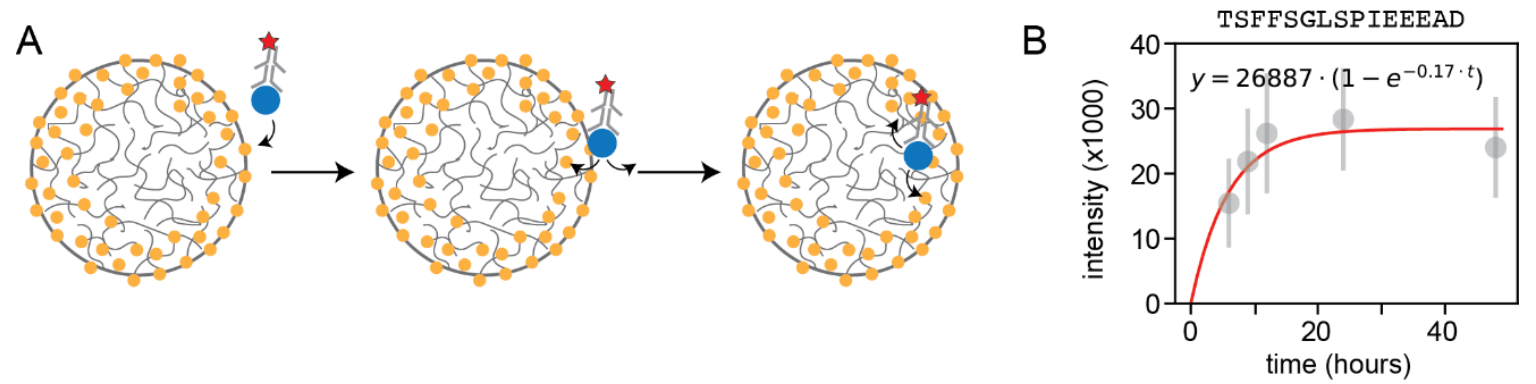

Figure 3. Hydrogel beads reduce dissociation rates to allow equilibrium binding measurements for even weak interactions. (A) Cartoon schematic showing proposed mechanism in which proteins binding to bead-bound peptides become trapped in bead hydrogel mesh. (B) Time course showing measured intensity as a function of time for B56-antibody complexes interacting with a peptide displayed by MRBLEs hydrogel beads. Markers indicate median intensities, error bars indicate standard deviation, and red line indicates a fit to kinetic binding model (equation and fitted parameters displayed at top of graph). 
bioRxiv preprint doi: https://doi.org/10.1101/2020.12.16.423088; this version posted December 16,2020 . The copyright holder for this preprint (which was not certified by peer review) is the author/funder, who has granted bioRxiv a license to display the preprint in perpetuity. It is made available under aCC-BY-NC-ND 4.0 International license.
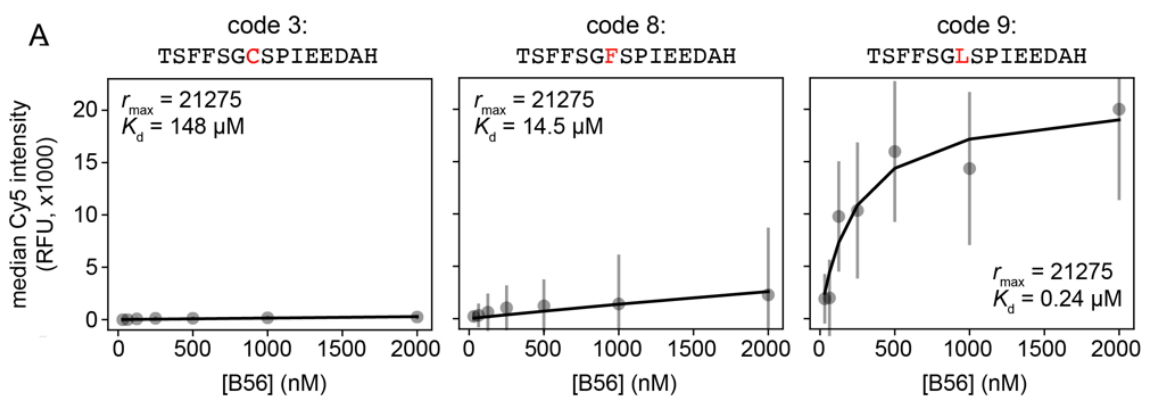

B

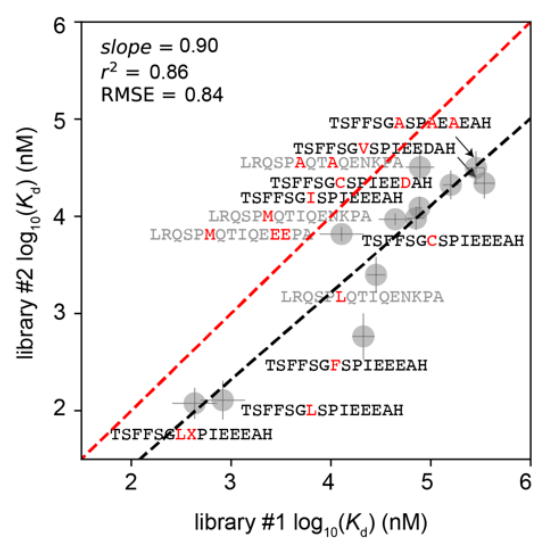

C

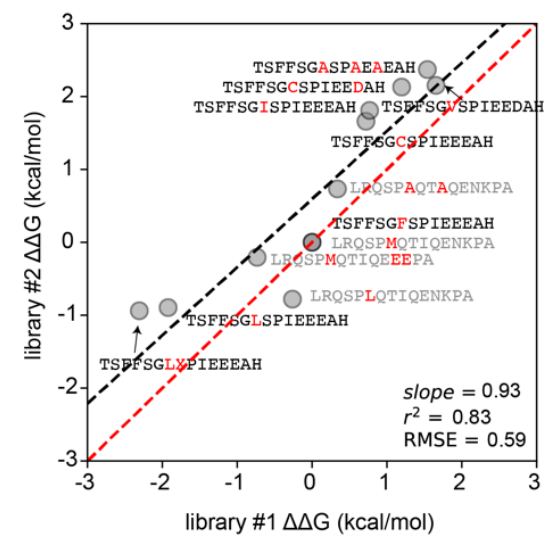

Figure 4. Technical reproducibility of MRBLE-pep replicate binding measurements. (A) Concentration-dependent binding data from library \#1 for B56 interacting with 3 peptides containing different single amino acid substitutions within the Kif4A LxxIxE SLiM. Grey markers indicate median fluorescence intensities for all MRBLEs displaying a particular peptide sequence at a given concentration; error bars indicate standard deviation of intensities. Solid grey lines denote Langmuir isotherm fits sharing a single maximum intensity saturation value (see Methods). (B) Measured $\log _{10}$-transformed binding affinities for 12 peptides measured via 2 independent full technical MRBLE-pep replicate experiments. Grey markers indicate the mean of 3 independent MRBLE-pep replicates for a given library; error bars denote the associated standard deviation. The red dashed line indicates a 1:1 relationship and the black dashed line indicates the results of a linear regression to the $\log _{10}$-transformed $K_{d}$ values. (C) Measured $\Delta \Delta G s$ for 12 peptides measured across 2 full technical MRBLE-pep replicates. Grey markers indicate the mean of 3 independent MRBLE-pep replicates for a given library; error bars denote the associated standard deviation. The red dashed line indicates a 1:1 relationship and the black dashed line indicates the results of a linear regression. 
bioRxiv preprint doi: https://doi.org/10.1101/2020.12.16.423088; this version posted December 16, 2020. The copyright holder for this preprint (which was not certified by peer review) is the author/funder, who has granted bioRxiv a license to display the preprint in perpetuity. It is made available under aCC-BY-NC-ND 4.0 International license.
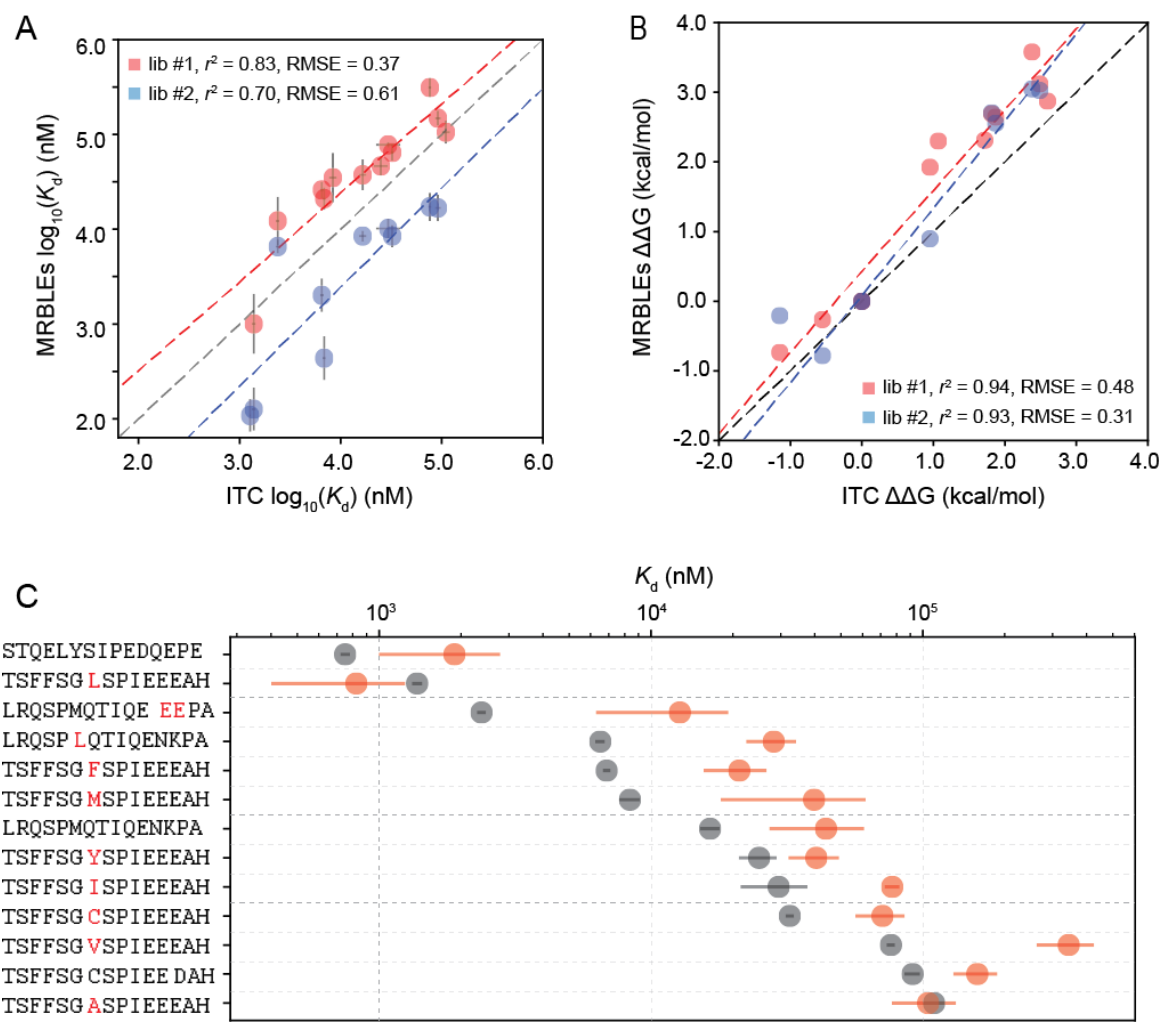

Figure 5. Comparison between MRBLE-pep and ITC affinity measurements. (A) Comparison of absolute affinities ( $\left.K_{d} S\right)$ measured via MRBLE-pep libraries \#1 (red) and \#2 (blue) and ITC (data from Hertz*, Kruse*, et al.) for 9 peptides derived from Kif4A and FoxO3 SLiM recognition motifs present in all 3 libraries (see Fig. 1D). Each MRBLE-pep measurement represents the median of 3 technical replicates and error bars indicate the standard error of the mean; for ITC measurements, error bars indicate the uncertainties returned from Langmuir isotherm fits. (B) Comparison of relative differences in affinity ( $\Delta \Delta G s)$ measured via MRBLE-pep libraries \#1 (red) and \#2 (blue) and ITC (data from Hertz*,Kruse*, et al.) for 9 peptides derived from Kif4A and FoxO3 SLiM recognition motifs present in all 3 libraries (see Fig. 1D). (C) Comparison between affinities measured by MRBLE-pep library \#1 (red) and ITC (data from (Hertz et al. 2016)) for 13 peptides present in both libraries. 


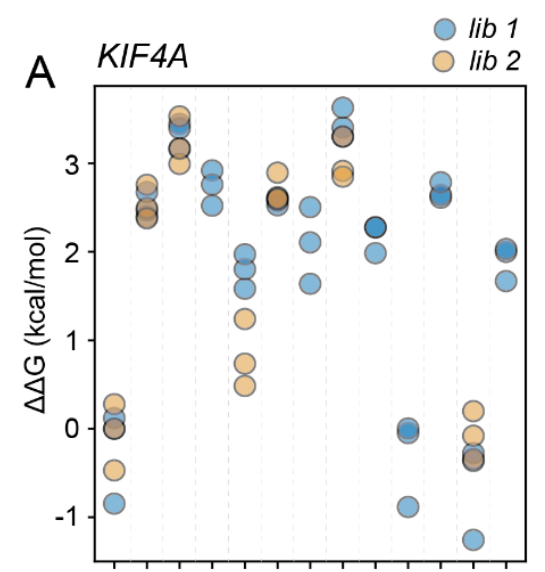

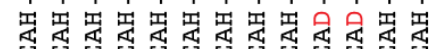

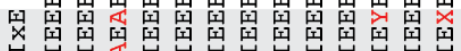

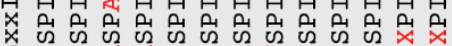

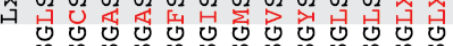

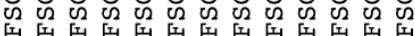

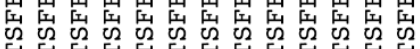
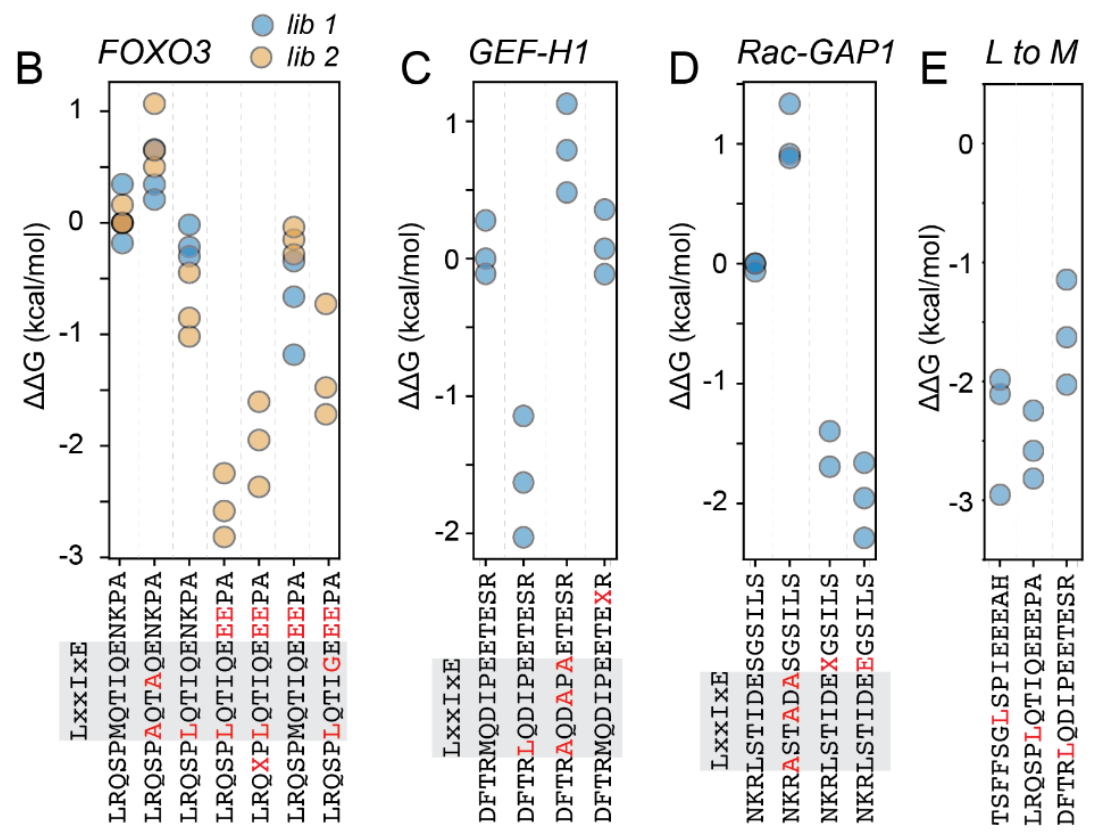

Figure 6. MRBLE-pep measurements quantifying the effects of systematic amino acid substitutions within the Kif4A, FoxO3, GEF$\mathrm{H} 1$, and Rac-GAP1 LxxIxE SLiM on B56 binding affinity. (A) Measured $\triangle \triangle$ Gs for systematic substitutions within the Kif4A LxxIxE SLiM relative to the median TSFFSGLSPIEEEAH value. (B) Measured $\triangle \Delta G$ s for systematic substitutions within the FoxO3 LxxIXE SLiM relative to the median WT (LRQSPMQTIQENKPA) value. (C) Measured $\triangle \triangle$ Gs for systematic substitutions within the GEF-H1 LxxIXE SLiM relative to the median WT (LDFTRMQDIPEETESR) value. (D) Measured $\triangle \Delta$ Gs for systematic substitutions within the Rac-GAP1 (NKRLSTIDESGSILS) value. (E) Measured $\triangle \Delta$ Gs for M to L substitutions within 3 different peptide backbones (Kif4A, FoxO3, and GEF$\mathrm{H} 1)$. 